PROCEEDINGS OF THE

AMERICAN MATHEMATICAL SOCIETY

Volume 131, Number 8, Pages 2585-2592

S 0002-9939(02)06789-8

Article electronically published on December 30, 2002

\title{
A PARTITION RELATION USING STRONGLY COMPACT CARDINALS
}

\author{
SAHARON SHELAH \\ (Communicated by Carl G. Jockusch, Jr.)
}

\begin{abstract}
If $\kappa$ is strongly compact and $\lambda>\kappa$ and $\lambda$ is regular (or alternatively $\operatorname{cf}(\lambda) \geq \kappa)$, then $\left(2^{<\lambda}\right)^{+} \rightarrow(\lambda+\zeta)_{\theta}^{2}$ holds for $\zeta, \theta<\kappa$.
\end{abstract}

\section{$\S 0$. INTRODUCTION}

The aim of this paper is to prove the following theorem.

0.1 Theorem. If $\kappa$ is a strongly compact cardinal, $\lambda>\kappa$ is regular and $\zeta, \theta<\kappa$, then the partition relation $\left(2^{<\lambda}\right)^{+} \rightarrow(\lambda+\zeta)_{\theta}^{2}$ holds.

0.2 Theorem. Assume the conditions in Theorem 0.1 hold, with " $\lambda$ regular". Then $\operatorname{cf}(\lambda)>\kappa$ suffices.

We notice that our argument is valid in the case $\kappa=\omega$. As for the history of the problem we point out that Hajnal proved, in an unpublished work, that $\left(2^{\omega}\right)^{+} \rightarrow\left(\omega_{1}+n\right)_{2}^{2}$ holds for every $n<\omega$. Then it was shown in [Sh:26], $\S 6$, that for $\kappa>\omega$ regular and $2^{|\alpha|}<\kappa$, the relation $\left(2^{<\kappa}\right)^{+} \rightarrow(\kappa+\alpha)_{2}^{2}$ is true. More recently Baumgartner, Hajnal, and Todorčević in BHT93] extended this to the case when the number of colors is arbitrarily finite. Earlier in [Sh:424], we have $\left(2^{<\lambda}\right)^{+n} \rightarrow$ $(\lambda \times m)_{k}^{2}$ for $n$ large enough (this was complimentary to the main result there that $\aleph_{0}<\lambda=\lambda^{<\lambda}+2^{\lambda}$ arbitrarily large does not imply $\left.2^{\lambda} \rightarrow(\lambda \times \omega)_{2}^{2}\right)$. Subsequently BHT93 improves $n$. We hope that the way the strong compactness was used will be useful elsewhere; see Sh:666 for a discussion of a possible consistency of failure. I also thank Peter Komjath for improving the presentation.

Notation. If $S$ is a set and $\kappa$ a cardinal, then $[S]^{\kappa}=\{a \subseteq S:|a|=\kappa\},[S]^{<\kappa}=\{a \subseteq$ $S:|a|<\kappa\}$. If $D$ is some filter over a set $S$, then $X \in D^{+}$denotes that $S \backslash X \notin D$ and $X \subseteq S$. If $\kappa<\mu$ are regular cardinals, then $S_{\kappa}^{\mu}=\{\alpha<\mu: \operatorname{cf}(\alpha)=\kappa\}$, a stationary set. The notation $A=\left\{x_{\alpha}: \alpha<\gamma\right\}<$, etc., means that $A$ is enumerated increasingly.

Received by the editors January 16, 2002 and, in revised form, March 12, 2002

2000 Mathematics Subject Classification. Primary 03E02.

The author thanks Alice Leonhardt for the beautiful typing.

Research of the author was partially supported by the United States-Israel Binational Science Foundation. Publication 761. 


\section{$\S 1$. THE CASE OF $\lambda$ REgulaR}

1.1 Lemma. Assume $\mu=\mu^{\theta}$. Assume that $D$ is a normal filter on $\mu^{+}$and $A^{*} \in D^{+}$satisfies $\delta \in A^{*} \Rightarrow c f(\delta)>\theta$, and $F^{\prime}$ is a function with domain $\left[A^{*}\right]^{2}$ and range of cardinality $\theta$. Then there are a normal filter $D_{0}$ on $\mu^{+}$extending $D, A_{0} \in D_{0}$ with $A_{0} \subseteq A^{*}$ and $C_{0} \subseteq \operatorname{Rang}\left(F^{\prime}\right)$ satisfying $\operatorname{Rang}\left(F^{\prime} \uparrow\left[A_{0}\right]^{2}\right)=C_{0}$ such that, if $X \in D_{0}^{+}$, then $\operatorname{Rang}\left(F^{\prime} \uparrow[X]^{2}\right) \supseteq C_{0}$.

We first prove a claim.

1.2 Claim. Assume $\mu=\mu^{\theta}$ and $F^{\prime}:\left[S^{*}\right]^{2} \rightarrow \theta, D$ is a normal filter on $\mu^{+}, S^{*} \subseteq \mu^{+}$ belongs to $D^{+}$and $\delta \in S^{*} \Rightarrow c f(\delta)>\theta$. There is a set $A \in D^{+}$such that $A \subseteq S^{*}$ and some $C \subseteq \theta$ satisfying $\operatorname{Rang}\left(F^{\prime} \uparrow[A]^{2}\right)=C$ and, if $f: A \rightarrow \mu^{+}$is a regressive function, then for some $\alpha<\mu^{+}$we have Rang $\left(F^{\prime}\left\lceil\left[f^{-1}(\alpha)\right]^{2}\right)=C\right.$ and $f^{-1}(\alpha)$ is a subset of $\mu^{+}$from $D^{+}$.

Proof. Toward contradiction assume that no such sets $A, C$ exist. We build a tree $T$ as follows. Every node $t$ of the tree will be of the form

$$
\begin{aligned}
t & =\left\langle\left\langle A_{\alpha}: \alpha \leq \varepsilon\right\rangle,\left\langle f_{\alpha}: \alpha<\varepsilon\right\rangle,\left\langle i_{\alpha}: \alpha<\varepsilon\right\rangle\right\rangle \\
& =\left\langle\left\langle A_{\alpha}^{t}: \alpha \leq \varepsilon\right\rangle,\left\langle f_{\alpha}^{t}: \alpha<\varepsilon\right\rangle,\left\langle i_{\alpha}^{t}: \alpha<\varepsilon\right\rangle\right\rangle
\end{aligned}
$$

for some ordinal $\varepsilon=\varepsilon(t)$ where $\left\langle A_{\alpha}: \alpha \leq \varepsilon\right\rangle$ is a decreasing, continuous sequence of subsets of $\mu^{+}$; for every $\alpha<\varepsilon, f_{\alpha}$ is a regressive function on $A_{\alpha}$; and $\left\langle i_{\alpha}: \alpha<\varepsilon\right\rangle$ is a sequence of distinct elements of $\theta$. It will always be true that if $t<_{T} t^{\prime}$, then each of the three sequences of $t^{\prime}$ extend the corresponding one of $t$.

To start, we make the node $t$ with $\varepsilon(t)=0, A_{0}=S^{*}$ the root of the tree.

At limit levels we extend (the obvious way) all cofinal branches to a node.

If we are given an element $t=\left\langle\left\langle A_{\alpha}: \alpha \leq \varepsilon\right\rangle,\left\langle f_{\alpha}: \alpha<\varepsilon\right\rangle,\left\langle i_{\alpha}: \alpha<\varepsilon\right\rangle\right\rangle$ of the tree and the set $A_{\varepsilon}$ is $=\emptyset \bmod D$, then we leave $t$ as a terminal node. Otherwise, let $C=C_{t}=\operatorname{Rang}\left(F^{\prime} \uparrow\left[A_{\varepsilon}\right]^{2}\right)$ and notice that by hypothesis, toward contradiction, the pair $A_{\varepsilon}, C_{t}$ cannot be as required in the Claim. There is, therefore, a regressive function $f=f_{t}$ with domain $A_{\varepsilon}$, such that for every $x<\mu^{+}$the set $\operatorname{Rang}\left(F^{\prime}\left\lceil\left[f^{-1}(x)\right]^{2}\right)\right.$ is a proper subset of $C_{t}$ or $f^{-1}(x)$ is a $=\emptyset \bmod D$ subset of $\mu^{+}$. We make as immediate extensions of $t$ the sequences of the form $t_{x}=$ $\left\langle\left\langle A_{\alpha}: \alpha \leq \varepsilon+1\right\rangle,\left\langle f_{\alpha}: \alpha<\varepsilon+1\right\rangle,\left\langle i_{\alpha}: \alpha<\varepsilon+1\right\rangle\right\rangle$ where $A_{\varepsilon+1}=f^{-1}(x), f_{\alpha}=f_{t}$ and $i_{\varepsilon} \in C_{t}$ is some colour value such that if $A_{\varepsilon+1} \neq \emptyset \bmod D$, then $i_{\varepsilon}$ is not in the range of $F^{\prime} \uparrow\left[A_{\varepsilon}\right]^{2}$.

Having constructed the tree, observe that every element $x \in S^{*} \subseteq \mu^{+}$belongs to a set $A_{\varepsilon(x)}^{t(x)}$ for some (unique) terminal node $t(x)$ of $T$. Also, $\varepsilon(x)<\theta^{+}\left(<\mu^{+}\right)$ holds by the selection of the $i_{\beta}$ 's as $\left\langle i_{\alpha}^{t(x)}: \alpha<\varepsilon(x)\right\rangle$ is a sequence of members of $\theta$ with no repetitions while $\theta$, the set of colours, has $\theta$ members. For some set $S \subseteq S^{*}$ of ordinals $x<\mu^{+}$which belong to $D^{+}$(by the normality of $D$ ), the value of $\varepsilon(x)$ is the same, say $\varepsilon$. For $x \in S$ we let $g_{\alpha}(x)=f_{\alpha}^{t(x)}(x)$ where $f_{\alpha}^{t(x)}$ is the $\alpha$-th regressive function in the node $t(x) \in T$. Again, by $\mu^{\theta}=\mu$ and $(\forall \alpha \in S)[\operatorname{cf}(\alpha)>\theta]$ we have that $\left(\forall x \in S^{\prime}\right)(\forall \alpha<\varepsilon) g_{\alpha}(x)=\beta_{\alpha}$ holds for some sequence $\left\langle\beta_{\alpha}: \alpha<\varepsilon\right\rangle$ and subset $S^{\prime} \subseteq S$ from $D^{+}$. But then we get that the set $S^{\prime}$ satisfies $x, y \in S^{\prime} \Rightarrow\left(A_{\alpha}^{t(x)}, f_{\alpha}^{t(x)}, i_{\alpha}^{t(x)}\right)=\left(A_{\alpha}^{t(y)}, f_{\alpha}^{t(y)}, i_{\alpha}^{t(y)}\right)$ for every $\alpha<\varepsilon$; we can prove this by induction on $\alpha$. We can then prove that $A_{\varepsilon}^{t(x)}=A_{\varepsilon}^{t(y)}$ for $x, y \in S^{\prime}$. 
We can conclude that $x, y \in S^{\prime} \Rightarrow t(x)=t(y)$, so $S^{\prime} \subseteq A_{\varepsilon(t)}^{t}$ for some terminal node $t$, but this latter set is in $D^{+}$, a contradiction.

Proof of Lemma 1.1. Apply Claim 1.2 with $S^{*}=A^{*}$ to get corresponding $(C, A)$. Define the ideal $I$ as follows. For $X \subseteq \mu^{+}$we let $X \in I$ iff there are a member $E$ of $D$ and a regressive function $f: X \cap A \rightarrow \mu^{+}$such that every $\operatorname{Rang}\left(F^{\prime} \uparrow\left[f^{-1}(\alpha)\right]^{2}\right)$ is a proper subset of $C$ or $f^{-1}(\alpha)$ is a $=\emptyset \bmod D$ subset of $\mu^{+}$.

Now:

1.3 Claim. I is a normal ideal on $\mu^{+}\left(\right.$and $\left.A^{*}=\mu^{+} \bmod I\right)$.

Proof. Straightforward.

Set $D_{0}$ to be the dual filter of $I$, let $A_{0}=A$ and let $C_{0}=C$; by Claim 1.2 we are done.

1.4 Remark. 1) If Lemma 1.1 holds for some $D_{0}, A_{0}, C_{0}$, then it holds for $D_{1}, A_{1}, C_{0}$ when the normal filter $D_{1}$ extends $D_{0}$, and $A_{1} \in D_{1}$ satisfies $A_{1} \subseteq A_{0}$.

2) If $D_{0}, A_{0}, C_{0}$ satisfy Lemma 1.5 , and $X \in D_{0}^{+}$, then $X$ contains a homogeneous set of order type $\lambda+1$ of color $\xi$ for every $\xi \in C_{0}$.

3) Lemma 1.1 is closely related to the proof in Sh:26], i.e. 5.1 there.

Proof of Theorem 0.1. Let $\mu=2^{<\lambda}$, and let $F:\left[\mu^{+}\right]^{2} \rightarrow \theta$ be a colouring. We apply Lemma 1.1 for $A^{*}=S_{\operatorname{cf}(\lambda)}^{\mu^{+}},(F=F, \theta, \mu=\mu)$ and $D$ the club filter. We shall write $F(\alpha, \beta)$ for $F(\{\alpha, \beta\})$ and 0 for $F(\alpha, \alpha)$.

We fix $A_{0}, D_{0}, C_{0}$ which we get by Lemma 1.1 .

1.5 Lemma. Almost every $\delta \in A_{0}$ (i.e. for all but a set $=\emptyset \bmod D_{0}$ ) satisfies the following: if $s \in\left[A_{0} \cap \delta\right]^{<\lambda}$ and $\left\{z_{\alpha}: \alpha<\gamma\right\}<\subseteq A_{0} \cap\left[\delta, \mu^{+}\right)$with $\gamma<\kappa$, then there is $\left\{y_{\alpha}: \alpha<\gamma\right\}<\subseteq A_{0} \cap(\sup (s), \delta)$ such that:

(a) $F\left(x, y_{\alpha}\right)=F\left(x, z_{\alpha}\right) \quad($ for $x \in s, \alpha<\gamma)$;

(b) $F\left(y_{\alpha}, y_{\beta}\right)=F\left(z_{\alpha}, z_{\beta}\right) \quad($ for $\alpha<\beta<\gamma)$.

Proof. By simple reflection (using the regularity of $\lambda$ ).

1.6 Lemma. Ther@ is $A_{0}^{\prime} \subseteq A_{0}, A_{0}^{\prime} \in D_{0}$ such that if $\delta \in A_{0}^{\prime}, s \in[\delta]^{<\lambda}$ and $\xi \in C_{0}$, then there exists a $\delta_{1} \in A_{0}, \delta<\delta_{1}$ such that:

(a) $F(x, \delta)=F\left(x, \delta_{1}\right) \quad($ for $x \in s)$;

(b) $F\left(\delta, \delta_{1}\right)=\xi$.

Proof. Otherwise, there is some $X \subseteq A_{0}, X \in D_{0}^{+}$such that for every $\delta \in X$ there are $s(\delta) \in[\delta]^{<\lambda}$ and $\xi(\delta) \in C_{0}$ such that there is no $\delta_{1}>\delta$ satisfying (a) and (b). By normality and $\mu=\mu^{<\lambda}$ we can assume that $s(\delta)=s$ and $\xi(\delta)=\xi$ holds for $\delta \in X$. By Lemma 1.1, that is, the choice of $\left(A_{0}, D_{0}, C_{0}\right)$, there must exist $\delta<\delta_{1}$ in $X$ with $F\left(\delta, \delta_{1}\right)=\xi$, and this is a contradiction.

Continuation of the proof of Theorem 0.1 . Let $A_{0}^{\prime} \subseteq A_{0}$ satisfy Lemmas 1.1 and 1.6 and pick some $\delta_{1} \in A_{0}^{\prime}$. Then let $T=A_{0}^{\prime} \backslash\left(\delta_{1}+1\right)$.

\footnotetext{
${ }^{1}$ In fact, if $A_{1}^{*} \in D_{0}^{+}$, then for some $A_{0}^{\prime} \subseteq A_{1} \cap A_{0}, A_{1} \backslash A_{0}^{\prime}=\emptyset$ modulo $D_{0}$ and the conclusion holds for every $\delta \in A_{0}^{\prime}$.
} 
1.7 Lemma. There exists a function $G: T \times T \rightarrow C_{0}$ such that if $s \in\left[\delta_{1}\right]^{<\lambda}, \gamma<\kappa$, and $Z=\left\{z_{\alpha}: \alpha<\gamma\right\}<\subseteq T$, then there is $\left\{y_{\alpha}: \alpha<\gamma\right\}<\subseteq\left(\sup (s), \delta_{1}\right)$ such that:

(a) $F\left(x, y_{\alpha}\right)=F\left(x, z_{\alpha}\right) \quad($ for $x \in s, \alpha<\gamma)$;

(b) $F\left(y_{\alpha}, y_{\beta}\right)=F\left(z_{\alpha}, z_{\beta}\right) \quad($ for $\alpha<\beta<\gamma)$;

(c) $F\left(y_{\alpha}, z_{\beta}\right)=G\left(z_{\alpha}, z_{\beta}\right) \quad($ for $\alpha, \beta<\gamma)$.

Proof. As $\kappa$ is strongly compact, it suffices to show that for every $Z \in[T]^{<\kappa}$ there exists a function $G: Z \times Z \rightarrow \theta$ as required. Clauses (a) and (b) are obvious by Lemma 1.5, and it is clear that, if we fix $Z$, then for every $s \in\left[\delta_{1}\right]^{<\lambda}$ there is an appropriate $G: Z \times Z \rightarrow \theta$. We show that there is some $G: Z \times Z \rightarrow \theta$ that works for every s. Assume otherwise, that is, for every $G: Z \times Z \rightarrow \theta$ there is some $s_{G} \in\left[\delta_{1}\right]^{<\lambda}$ such that $G$ is not appropriate for $s_{G}$. Notice that the number of these functions $G$ is less than $\kappa$. Then no $G$ could be right for $s=\bigcup\left\{s_{G}: G\right.$ a function from $Z \times Z$ to $\theta\} \in\left[\delta_{1}\right]^{<\lambda}$, a contradiction.

Continuation of the proof of Theorem 0.1. We now apply Lemma 1.1 to the colouring $\bar{G}\{x, y\}=\bar{G}(x, y)=\langle F(x, y), G(x, y)\rangle$ for $x<y$ in $T$ and 0 otherwise, and the filter $D_{0}$ and the set $T$ to get the normal filter $D_{1} \supseteq D_{0}$, the set $A_{1} \subseteq T \subseteq A_{0}^{\prime}$ such that $A_{1} \in D_{1}$ and the colour set $C_{1} \subseteq \theta \times \theta$. Notice that actually $C_{1} \subseteq C_{0} \times C_{0}$. We can also apply Lemmas 1.5 and 1.6 to get some set $A_{1}^{\prime} \subseteq A_{1}$.

1.8 Lemma. There is a set $a \in\left[A_{1}^{\prime}\right]^{<\kappa}$ such that for every decomposition $a=$ $\bigcup\left\{a_{\bar{\xi}}: \bar{\xi} \in C_{1}\right\}$ there is some $\bar{\xi} \in C_{1}$ such that:

( $\alpha$ ) for every $\bar{\varepsilon} \in C_{1}$ there is an $\bar{\varepsilon}$-homogeneous subset for the colouring $\bar{G}$ of order type $\zeta$ in $a_{\bar{\xi}}$;

$(\beta)$ similarly for every $\varepsilon \in C_{0}$ and $F$.

Proof. This follows from the strong compactness of $\kappa$, as $A_{1}^{\prime}$ itself has this partition property (see Claim 2.8 for more details).

Continuation of the proof of Theorem 0.1. Fix a set $a$ as in Lemma 1.8.

We now describe the construction of the required homogeneous subset. Let $\delta_{2} \in A_{1}^{\prime}$ be some element with $\delta_{2}>\sup (a)$. For $\bar{\xi}=\left(\xi_{1}, \xi_{2}\right) \in C_{1} \subseteq \theta \times \theta$ let $a_{\bar{\xi}}$ be the following set:

$$
a_{\bar{\xi}}=\left\{x \in a: \bar{G}\left(x, \delta_{2}\right)=\bar{\xi}\right\} .
$$

By Lemma 1.8, there is some $\bar{\xi}=\left(\xi_{1}, \xi_{2}\right) \in C_{1}$ for which the statement in that lemma is true and necessarily (as $a \cup\left\{\delta_{2}\right\} \subseteq A_{1}^{\prime} \subseteq A_{0}$ and $a_{\bar{\xi}} \neq \emptyset$ ) we have $\xi_{1}, \xi_{2} \in C_{0}$. Select some $b \subseteq a_{\bar{\xi}}$, otp $(b)=\zeta$ such that $F$ is constantly $\xi_{2}$ on $b$; this is possible by clause $(\beta)$ of Lemma 1.8. This set $b$ will be the $\zeta$ part of our homogeneous set of ordinals of order type $\lambda+\zeta$, so we will have to construct a set of order type $\lambda$ below $b$. By induction on $\alpha$ we will choose $x_{\alpha}$ such that the set $\left\{x_{\alpha}: \alpha<\lambda\right\}<\subseteq \delta_{1}$ satisfies the following conditions:

$(*)_{1} \quad F\left(x_{\beta}, x_{\alpha}\right)=\xi_{2}$ (for $\left.\beta<\alpha\right)$,

$(*)_{2} F\left(x_{\alpha}, b \cup\left\{\delta_{2}\right\}\right)=\xi_{2}$, i.e. $F\left(x_{\alpha}, y\right)=\xi_{2}$ when $y \in b \cup\left\{\delta_{2}\right\}$.

Assume that we have reached step $\alpha$, that is, we are given the set of ordinals with $\left\{x_{\beta}: \beta<\alpha\right\}_{<}$and call this set $s$. Applying Lemma 1.6 for $A_{1}, A_{1}^{\prime}, \delta_{2}$ and $s \cup b$ and the colouring $\bar{G}$ here standing for $A_{0}, A_{0}^{\prime}, \delta, s$ and the colouring $F$ there (that is, the choice of $A_{1}^{\prime}$ ) we get that there exists some $\delta_{3}>\delta_{2}$ (standing for $\delta_{1}$ there) 
such that:

(i) $\delta_{3} \in A_{1}$;

(ii) $\bar{G}\left(x, \delta_{3}\right)=\bar{G}\left(x, \delta_{2}\right)$ for $x \in s \cup b$;

(iii) $\bar{G}\left(\delta_{2}, \delta_{3}\right)=\left(\xi_{1}, \xi_{2}\right)$.

Hence

$(*)_{3} F\left(x_{\beta}, \delta_{3}\right)=\xi_{2}($ for $\beta<\alpha)$.

[Why? As $F\left(x_{\beta}, \delta_{3}\right)=F\left(x_{\beta}, \delta_{2}\right)$ by (ii) and the choice of $\bar{G}$ and $F\left(x_{\beta}, \delta_{2}\right)=$ $\xi_{2}$ by $(*)_{2}$ from the induction hypothesis.]

$(*)_{4} G\left(b \cup\left\{\delta_{2}\right\}, \delta_{3}\right)=\xi_{2}$, i.e. $G\left(y, \delta_{3}\right)=\xi_{2}$ when $y \in b \cup\left\{\delta_{2}\right\}$.

[Why? If $y \in b$, then by (ii) and the definition of $\bar{G}$ we have $G\left(y, \delta_{3}\right)=$ $G\left(y, \delta_{2}\right)$, but $b \subseteq a_{\bar{\xi}}$ so by the choice of $a_{\bar{\xi}}$ we have $G\left(y, \delta_{2}\right)=\xi_{2}$. For $y=\delta_{2}$ use clause (iii), that is, $\left(\xi_{1}, \xi_{2}\right)=\bar{G}\left(\delta_{2}, \delta_{3}\right)=\left(F\left(\delta_{2}, \delta_{3}\right), G\left(\delta_{2}, \delta_{3}\right)\right)$.]

By the choice of $G$ this implies that there is some $x_{\alpha}$ as required; that is, by the choice of $\bar{G}$ (see Lemma 1.7) applied to $Z=\left\{z_{i}: i<\gamma\right\}$, enumerating the set $b \cup\left\{\delta_{2}, \delta_{3}\right\}$ and $s$ as above, we get $\left\{y_{i}: i<\gamma\right\}$, now necessarily $\delta_{3}=z_{\gamma-1}$, and we can choose $y_{\gamma-1}$ as $x_{\alpha}$.

\section{$\S 2$. ThE CASE OF $\lambda$ SINGUlaR}

We prove version 0.2 of the main theorem.

Proof of Theorem 0.2. Let $\sigma=\operatorname{cf}(\lambda)$. Let $\lambda=\sum_{\varepsilon<\sigma} \lambda_{\varepsilon}$ with $\lambda_{\varepsilon}>\sigma \geq \kappa>\theta$ strictly increasing. Let $\mu_{\varepsilon}=2^{\lambda_{\varepsilon}}$ and $\mu=\Sigma\left\{\mu_{\varepsilon}: \varepsilon<\sigma\right\}=2^{<\lambda}$. We also fix $F:\left[\mu^{+}\right]^{2} \rightarrow \theta$.

2.1 Claim. For some $\overline{\mathcal{C}}$ we have:

(a) $\overline{\mathcal{C}}=\left\langle\mathcal{C}_{\alpha}: \alpha \in S\right\rangle$;

(b) $S \subseteq \mu^{+}, \mathcal{C}_{\delta} \subseteq \delta$;

(c) $\operatorname{otp}\left(\mathcal{C}_{\delta}\right) \leq \sigma$;

(d) $S^{*}=\left\{\delta<\lambda: \operatorname{otp}\left(\mathcal{C}_{\delta}\right)=\sigma\right\}$ is stationary;

(e) $\mathcal{C}_{\delta}$ unbounded in $\delta$ if $\operatorname{otp}\left(\mathcal{C}_{\delta}\right)=\sigma$;

(f) $\alpha \in \mathcal{C}_{\delta} \Rightarrow \alpha \in S$ and $\mathcal{C}_{\alpha}=\mathcal{C}_{\delta} \cap \alpha$.

Proof. By [Sh:420, §1] as $\sigma^{+}<\mu^{+}, \sigma=\operatorname{cf}(\sigma)$.

Continuation of the proof of Theorem 0.2 . Let $D_{0}, A_{0}, C_{0}$ be as given by Lemma 1.1 with the club filter of $\mu^{+}, S^{*}$ (from clause (d) of Claim 2.1 above) here standing for $D, A^{*}$ there, so $A_{0} \subseteq S^{*}$.

Notation. $\varepsilon(\alpha)=\operatorname{otp}\left(\mathcal{C}_{\alpha}\right)$.

2.2 Claim. Let $\chi>2^{\mu},<_{\chi}^{*}$ a well ordering of $\mathcal{H}(\chi)$ ). For any $x \in \mathcal{H}(\chi)$ we can find $\overline{\mathfrak{B}}=\left\langle\mathfrak{B}_{\alpha}: \alpha<\lambda\right\rangle$ such that:

(a) $\mathfrak{B}_{\alpha} \prec\left(\mathcal{H}(\chi), \in,<_{\chi}^{*}\right)$;

(b) $\bar{\lambda}, \mu, F,\left\langle\lambda_{\varepsilon}: \varepsilon<\sigma\right\rangle, \overline{\mathcal{C}}, A_{0}, C_{0}, D_{0}$ belong to $\mathfrak{B}_{\alpha}$;

(c) $\left\langle\mathfrak{B}_{\beta}: \beta<\alpha\right\rangle \in \mathfrak{B}_{\alpha}$ if $\alpha \notin S^{*}$;

(d) $\left\|\mathfrak{B}_{\beta}\right\|=\mu_{\varepsilon(\beta)}$ and $\left[\mathfrak{B}_{\beta}\right] \leq \lambda_{\varepsilon(\beta)} \subseteq \mathfrak{B}_{\beta}$ and $\mu_{\varepsilon(\beta)}+1 \subseteq \mathfrak{B}_{\beta}$ (actually follows);

(e) $\mathfrak{B}_{\alpha}=\bigcup\left\{\mathfrak{B}_{\beta}: \beta \in \mathcal{C}_{\alpha}\right\}$ if $\alpha \in S^{*}$.

Proof. Straightforward. 
2.3 Observation. 1) We have $\varepsilon(\alpha)<\varepsilon(\beta)$, and $\mathfrak{B}_{\alpha} \in \mathfrak{B}_{\beta}$ and $\mathfrak{B}_{\alpha} \prec \mathfrak{B}_{\beta}$ if $\alpha \in \mathcal{C}_{\beta}$.

2.4 Claim. There is a set $A_{0}^{\prime} \subseteq A_{0}$ such that:

( $\alpha) A_{0}^{\prime} \in D_{0}$ and $\alpha<\delta \in A_{0}^{\prime} \Rightarrow \sup \left(\mathfrak{B}_{\alpha} \cap \mu^{+}\right)<\delta$;

( $\beta)$ if $\xi \in C_{0}$ and $\delta \in A_{0}^{\prime}$ and $s \in \bigcup\left\{\left[\delta \cap \mathfrak{B}_{\alpha}\right] \leq \lambda_{\varepsilon(\alpha)}: \alpha \in \mathcal{C}_{\delta}\right\}$, then there is $\delta_{1} \in A_{0}$ such that $\delta<\delta_{1}$ and

(a) $F(x, \delta)=F\left(x, \delta_{1}\right)$ for $x \in s$,

(b) $F\left(\delta, \delta_{1}\right)=\xi$.

Proof. Requirement $(\alpha)$ holds for all but a nonstationary set of $\delta \in A_{0}$. Requirement $(\beta)$ is proved as in Lemma 1.6.

Now fix $A_{0}^{\prime} \subseteq A_{0}$ as in Claim 2.4, and fix $\delta_{1} \in A_{1}^{\prime}$ and let $T=A_{0}^{\prime} \backslash\left(\delta_{1}+1\right)$. Recall $\delta_{1} \in A_{0}^{\prime} \subseteq S^{*}=\left\{\delta: \operatorname{otp}\left(\mathcal{C}_{\delta}\right)=\sigma, \delta=\sup \left(\mathcal{C}_{\delta}\right)\right\} \subseteq\left\{\delta<\mu^{+}: \operatorname{cf}(\delta)=\sigma\right\}$.

2.5 Claim. There is a function $G_{\varepsilon}: T \times T \rightarrow C_{0}$ such that:

$\square$ if $s \in\left[\delta \cap \mathfrak{B}_{\alpha}\right] \leq \lambda_{\varepsilon}$ and $\varepsilon=\varepsilon(\alpha)$ and $\alpha \in \mathcal{C}_{\delta_{1}}$ and $\gamma<\kappa$ and $Z=\left\{z_{\beta}: \beta<\right.$ $\gamma\}<\subseteq T$, then there is $\left\{y_{\beta}: \beta<\gamma\right\}<\subseteq \delta \cap \mathfrak{B}_{\alpha}=\mu^{+} \cap \mathfrak{B}_{\alpha}, y_{0}>\sup (s)$ such that:

(a) $F\left(x, y_{\beta}\right)=F\left(x, z_{\beta}\right)$ for $x \in s, \beta<\delta$;

(b) $F\left(z_{\beta_{1}}, y_{\beta_{2}}\right)=G\left(y_{\beta_{1}}, y_{\beta_{2}}\right)$;

(c) $F\left(z_{\beta_{1}}, z_{\beta_{2}}\right)=F\left(y_{\beta_{1}}, y_{\beta_{2}}\right)$ for $\beta_{1}<\beta_{2}<\gamma$.

Proof. As in Claim 1.7.

2.6 Claim. There exists a function $G: T \times T \rightarrow C_{0}$ such that if $s \in[T]^{<\kappa}$, then for arbitrarily large $\varepsilon<\sigma$ we have $G \uparrow(s \times s)=G_{\varepsilon} \uparrow(s \times s)$.

Proof. Let $D^{*}$ be a uniform $\kappa$-complete ultrafilter on $\sigma$ and define $G$ by $G(\alpha, \beta)$ is the unique $\xi \in C_{0}$ such that $\left\{\varepsilon<\sigma: G_{\varepsilon}(\alpha, \beta)=\xi\right\} \in D^{*}$.

Continuation of the proof of Theorem 0.2 . Now we apply Lemma 1.1 to the colouring $\bar{G}$ where $\bar{G}\{x, y\}=\bar{G}(x, y)=(F(x, y), G(x, y))$ for $x<y$ in $T$ and zero otherwise and to the filter $D_{0}$ and the set $T$. We get a normal filter $D_{1}$ and a set $A_{1} \subseteq T \subseteq A_{0}^{\prime}$ and a set of colours $C_{1}$. As $A_{1} \subseteq A_{0}$ necessarily $C_{1} \subseteq C_{0} \times C_{0}$.

2.7 Claim. There is $A_{1}^{\prime} \subseteq A_{1}$ such that:

( $\alpha) A_{1} \backslash A_{1}^{\prime}=\emptyset \bmod D_{1}$;

( $\beta)$ if $\delta \in A_{1}^{\prime}, \alpha \in \mathcal{C}_{\delta}$ and $s \in\left[\delta \cap \mathfrak{B}_{\alpha}\right]^{\leq \lambda_{\varepsilon(\alpha)}}$ and $\bar{\xi} \in C_{1}$, then for some $\delta_{*}$ we have $\delta<\delta_{*} \in A_{1}$ and

(a) $\bar{G}(x, \delta)=\bar{G}\left(x, \delta_{1}\right)$ for every $x \in s$,

(b) $\bar{G}\left(\delta, \delta_{*}\right)=\bar{\xi}$.

Proof. As in the proof of Lemma 1.6.

2.8 Claim. There is a set $a \in\left[A_{1}^{\prime}\right]^{<\kappa}$ such that:

$\square$ for every decomposition of a as $\bigcup\left\{a_{\bar{\xi}}: \bar{\xi} \in C_{1}\right\}$ there is $\bar{\xi} \in C_{1}$ such that:

$(\alpha)$ for every $\bar{\varepsilon} \in C_{1}$ there is $b \subseteq a_{\bar{\xi}}$ of order type $\zeta$ such that $\bar{G} \uparrow[b]^{2}$ is constantly $\bar{\varepsilon}$;

( $\beta$ ) for every $\varepsilon \in C_{0}$ there is $b \subseteq a_{\bar{\xi}}$ of order type $\zeta$ such that $F \uparrow[b]^{2}$ is constantly $\varepsilon$. 
Proof. The claim holds since $A_{1}^{\prime}$ has this property and $\kappa$ is strongly compact. If $A_{1}^{\prime}=\cup\left\{a_{\bar{\xi}}: \bar{\xi} \in C_{1}\right\}$ for some $\bar{\xi}, a_{\bar{\xi}} \in D_{1}^{+}$hence clause $(\alpha)$ holds by the choice of $D_{1}, C_{1}$; and clause $(\beta)$ holds as $D_{1}^{+} \subseteq D_{0}^{+}$(as $D_{0} \subseteq D_{1}$ ) and the choice of $D_{0}, C_{0}$.

Continuation of the proof of Theorem 0.2 . Now choose $\delta_{2} \in A_{1}^{\prime}$ such that $\delta_{2}>$ $\sup (a)$ and for $\bar{\xi}=\left(\xi_{1}, \xi_{2}\right) \in C_{1} \subseteq \theta \times \theta$ define $a_{\bar{\xi}}$ as

$$
\bar{a}_{\bar{\xi}}=\left\{x \in a: \bar{G}\left(x, \delta_{2}\right)=\bar{\xi}\right\} .
$$

Clearly $\left\langle a_{\bar{\xi}}: \bar{\xi} \in C_{1}\right\rangle$ is a decomposition of $a$ and so there is $\bar{\xi}=\left(\xi_{1}, \xi_{2}\right) \in C_{1}$ as guaranteed by $\square$ of Claim 2.8. In particular, there is $b \subseteq a_{\bar{\xi}}$ of order type $\zeta$ such that $F\left\lceil[b]^{2}\right.$ is constantly $\xi_{2}$ (note that $\left(\xi_{1}, \xi_{2}\right) \in C_{1} \subseteq C_{0} \times C_{0}$ so $\xi_{2} \in C_{0}$ ). Now let $E=\left\{\varepsilon<\sigma: G_{\varepsilon}\left(\alpha, \delta_{2}\right)=G\left(\alpha, \delta_{2}\right)\right.$ for every $\left.\alpha \in b\right\}$. By the definition of $G$ this is an unbounded subset of $\sigma$ and clearly

(*) if $\varepsilon \in E$ and $\alpha \in b$, then $G_{\varepsilon}\left(\alpha, \delta_{2}\right)=G\left(\alpha, \delta_{2}\right)=\left(\xi_{1}, \xi_{2}\right)$.

For $\alpha<\lambda$ let $\Upsilon(\alpha)=\operatorname{Min}\left\{\varepsilon \in E: \alpha<\lambda_{\varepsilon}\right\}$ and let $C_{\delta_{1}}=\{\gamma(\Upsilon): \Upsilon<\sigma\}_{<}$. Now we try to choose by induction on $\alpha<\lambda$ a element $x_{\alpha}$ satisfying

$(*)_{0} \quad x_{\alpha}<\delta_{1}$ and moreover $x_{\alpha} \in \delta_{1} \cap \mathfrak{B}_{\gamma(\Upsilon(\alpha))}$, and $\beta<\alpha \Rightarrow x_{\beta}<x_{\alpha}$,

$(*)_{1} F\left(x_{\beta}, x_{\alpha}\right)=\xi_{2}$ for $\beta<\alpha$,

$(*)_{2} F\left(x_{\alpha}, \beta\right)=\xi_{2}$ for $\beta \in b \cup\left\{\delta_{2}\right\}$.

At step $\alpha$, by Claim 2.7, that is, by the choice of $A_{1}^{\prime}$ applying clause $(\beta)$ there with $\left\{x_{\beta}: \beta<\alpha\right\} \cup b, \delta_{2}, \bar{\xi}$ here standing for $s, \delta, \bar{\xi}$ there, we can find $\delta_{3}$ satisfying the requirement there on $\delta_{1}$, so

(i) $\delta_{2}<\delta_{3} \in A_{1}$,

(ii) $\bar{G}\left(x, \delta_{3}\right)=\bar{G}\left(x, \delta_{2}\right)$ for $x \in s \cup b$,

(iii) $\bar{G}\left(\delta_{2}, \delta_{3}\right)=\left(\xi_{1}, \xi_{2}\right)$.

Now

$(*)_{3} \quad F\left(x_{\beta}, \delta_{3}\right)=\xi_{2}$ for $\beta<\alpha$.

[Why? By (ii) we have $\bar{G}\left(x_{\beta}, \delta_{3}\right)=\bar{G}\left(x_{\beta}, \delta_{2}\right)$, hence $F\left(x_{\beta}, \delta_{3}\right)=F\left(x_{\beta}, \delta_{2}\right)$, but the latter by $(*)_{2}$ is equal to $\xi_{2}$.]

$(*)_{4} G\left(\beta, \delta_{3}\right)=\xi_{2}$ for $\beta \in b$.

[Why? By (ii) and as $\left.\beta \in b \Rightarrow \bar{G}\left(\beta, \delta_{2}\right)=\left(\xi_{1}, \xi_{2}\right) \Rightarrow G\left(\beta, \delta_{2}\right)=\xi_{2}\right)$.]

$(*)_{5} G\left(\delta_{2}, \delta_{3}\right)=\xi_{2}$.

[Why? By clause (iii).]

$(*)_{6}\left\{x_{\beta}: \beta<\alpha\right\}$ is a subset of $\delta_{1} \cap \mathfrak{B}_{\gamma(\Upsilon(\alpha))}$.

Let $\left\langle y_{i}: i<\zeta+2\right\rangle$ list $b \cup\left\{\delta_{2}, \delta_{3}\right\}$ increasing order.

Now we use the choice of $G_{\Upsilon(\alpha)}$ to choose an increasing sequence $\left\langle z_{i}: i<\zeta+2\right\rangle$ in $\delta_{1} \cap \mathfrak{B}_{\gamma(\Upsilon(\alpha))}, z_{0}>x_{\beta}$ for $\beta<\alpha$ such that $F\left(z_{i}, y_{j}\right)=G\left(y_{i}, y_{j}\right)$ for $i, j<\zeta+2$ and $F\left(x_{\beta}, z_{i}\right)=F\left(x_{\beta}, y_{i}\right)$ for $i<\zeta+2$. Let $x_{\alpha}=z_{\zeta+1}$ so $x_{\alpha}=\delta_{1} \cap \mathfrak{B}_{\gamma(\Upsilon(\alpha))}$ is $>x_{\beta}$ for $\beta<\alpha$.

Also $x_{\alpha}$ satisfies $(*)_{0}$ of the recursive definition. Now $\beta<\alpha \Rightarrow F\left(x_{\beta}, x_{\alpha}\right)=$ $F\left(x_{\beta}, z_{\zeta+1}\right)=F\left(x_{\beta}, y_{\zeta+1}\right)=F\left(x_{\beta}, \delta_{3}\right)$ which is $\xi_{2}$ by $(*)_{3}$ above, so for our choice of $x_{\alpha},(*)_{1}$ holds. Next if $\beta \in b \cup\left\{\delta_{2}\right\}$, then $F\left(x_{\alpha}, x_{\beta}\right)=F\left(x_{\beta}, z_{\zeta+1}\right)=G\left(x_{\beta}, \delta_{3}\right)$ which is $\xi_{2}$ by $(*)_{4}$ or $(*)_{5}$. So $x_{\alpha}$ is as required. 


\section{REFERENCES}

[ERDHAJ] P. Erdős, A. Hajnal: Unsolved problems in set theory, Proc. Symp. Pure Math. 8, Providence, Rhode Island, 17-48. MR 43:6101

[BHT93] James Baumgartner, Andras Hajnal, and Stevo Todorčević. Extensions of the ErdosRado Theorems. In Finite and Infinite Combinatorics in Set Theory and Logic, pages 1-18. Kluwer Academic Publishers, 1993. N.W. Sauer et. al. eds. MR 95c:03111

[Sh:26] Saharon Shelah. Notes on combinatorial set theory. Israel Journal of Mathematics, 14:262-277, 1973. MR 48:5864

[Sh:420] Saharon Shelah. Advances in Cardinal Arithmetic. In Finite and Infinite Combinatorics in Sets and Logic, pages 355-383. Kluwer Academic Publishers, 1993. N.W. Sauer et al (eds.). MR 95h:03112

[Sh:424] Saharon Shelah. On $C H+2^{\aleph_{1}} \rightarrow(\alpha)_{2}^{2}$ for $\alpha<\omega_{2}$. In Logic Colloquium'90. ASL Summer Meeting in Helsinki, volume 2 of Lecture Notes in Logic, pages 281-289. Springer Verlag, 1993. math.LO/9308212 MR 95m:03098

[Sh:666] Saharon Shelah. On what I do not understand (and have something to say). Fundamenta Mathematicae, 166:1-82, 2000. math.LO/9906113 MR 2002a:03091

Institute of Mathematics, The Hebrew University, Jerusalem, Israel - and - Department of Mathematics, Rutgers University, New Brunswick, New Jersey

E-mail address: shelah@math.huji.ac.il

${ }^{2}$ References of the form math.xx/... refer to the xxx.lanl.gov archiv. 